\title{
Commuting elements and spaces of homomorphisms
}

\author{
Alejandro Adem • Frederick R. Cohen
}

Published online: 3 September 2009

(C) Springer-Verlag 2009

Erratum to: Math. Ann. (2007) 338:587-626 DOI 10.1007/s00208-007-0089-z

We correct some cohomology calculations appearing in [1].

In the preprint [2], M. Crabb has pointed out that two of the cohomology calculations in [1], namely Theorems 1.4 and 4.12 are not consistent with the results in his paper. Here we outline how to correct the results in our paper.

Consider the Mayer-Vietoris spectral sequence in integral cohomology associated to the covering $X[3]=X_{1,2} \cup X_{1,3} \cup X_{2,3}$ where $X$ [3] denotes the non-commuting triples in $S U(2)$ and the $X_{i, j}$ correspond to the non-commutativity of the $i$ th and $j$ th coordinates. It will have as its $E_{1}$ term

$E_{1}^{p, q} \cong \begin{cases}H^{q}\left(X_{1,2}, \mathbb{Z}\right) \oplus H^{q}\left(X_{1,3}, \mathbb{Z}\right) \oplus H^{q}\left(X_{2,3}, \mathbb{Z}\right) & \text { if } p=0 \\ H^{q}\left(X_{1,2} \cap X_{1,3}, \mathbb{Z}\right) \oplus H^{q}\left(X_{1,2} \cap X_{2,3}, \mathbb{Z}\right) \oplus H^{q}\left(X_{1,3} \cap X_{2,3}, \mathbb{Z}\right) & \text { if } p=1 \\ H^{q}\left(X_{123}, \mathbb{Z}\right) & \text { if } p=2 \\ 0 & \text { if } p \geq 3 .\end{cases}$

The online version of the original article can be found under doi:10.1007/s00208-007-0089-z.

Alejandro Adem was partially supported by NSERC and NSF.

Frederick R. Cohen was partially supported by DARPA and the NSF.

\footnotetext{
A. Adem (ه)

Department of Mathematics, University of British Columbia, Vancouver, BC V6T 1Z2, Canada e-mail: adem@math.ubc.ca

F. R. Cohen

Department of Mathematics, University of Rochester, Rochester, NY 14627, USA

e-mail: cohf@math.rochester.edu
} 
The differentials on the $E_{1}$-page are determined as alternating sums of the maps induced by inclusions. The cohomology of the spaces $X_{i, j}$ and their intersections were computed in [1]; there are homotopy equivalences

$$
X_{j, k} \simeq S O(3) \times S U(2), \quad X_{j, k} \cap X_{i, k} \simeq S O(3) \times \mathbb{S}^{1}, \quad X_{123} \simeq S O(3) \times\left[\mathbb{S}^{1} \bigvee \mathbb{S}^{1} \bigvee \mathbb{S}^{1}\right]
$$

The spectral sequence begins with $d_{1}^{1, q}: E_{1}^{i, q} \rightarrow E_{1}^{i+1, q}$ for $i=0,1,2$. These differentials can be computed explicitly, using our knowledge of these spaces and their intersections. First we must make a digression to consider the fundamental group.

By projecting $X_{1,2} \cap X_{1,3}$ onto its first two coordinates we obtain a split fibration onto the non-commuting pairs $X[2] \simeq S O$ (3) where the fiber is homotopic to $\mathbb{S}^{1}$. This product can be used to represent the generators of its fundamental group geometrically. Now consider the projection onto the first and third coordinates; again this yields a map onto $X$ [2]. However, the previous fiber, homotopic to the circle, is now mapped homotopically non-trivially, as it can be identified with the fiber of the projection of $X[2]$ onto $S U(2)-\{ \pm 1\}$ (which is simply further projection onto the first coordinate). Thus, what we have shown is that if $\pi_{1}\left(X_{12} \cap X_{13}\right) \cong \mathbb{Z} w \oplus \mathbb{Z} / 2 y$ with these generators corresponding to the geometric decomposition above, then the inclusions into $X_{12}$ and $X_{13}$ induce maps $f_{1}, f_{2}: \mathbb{Z} w \oplus \mathbb{Z} / 2 y \rightarrow \mathbb{Z} / 2 v$ such that $f_{1}(w)=0, f_{1}(y)=v$ and $f_{2}(w)=v, f_{2}(y)=v$. An immediate consequence of this is that $X$ [3] is simply connected, as can be seen by using the Seifert-Van Kampen Theorem for the same decomposition we are considering; indeed in $\pi_{1}(X[3])$ the homomorphisms induced by the inclusions of the intersections force the identification of the three generators but they also identify $f_{1}(w)=0$ with $f_{2}(w)=v$ and thus the fundamental group is trivial.

Back to the calculation: the key ingredients are that $E_{2}^{1, i}=0$ for all $i \geq 0$ and that

$$
E_{2}^{2, i} \cong\left\{\begin{array} { l l } 
{ 0 } & { \text { if } i = 0 } \\
{ \mathbb { Z } / 2 } & { \text { if } i = 1 } \\
{ 0 } & { \text { if } i = 2 } \\
{ \mathbb { Z } / 2 } & { \text { if } i = 3 } \\
{ \mathbb { Z } / 2 } & { \text { if } i = 4 } \\
{ 0 } & { \text { if } i \geq 5 }
\end{array} \quad E _ { 2 } ^ { 0 , i } \cong \left\{\begin{array}{ll}
\mathbb{Z} & \text { if } i=0 \\
0 & \text { if } i=1 \\
\mathbb{Z} / 2 & \text { if } i=2 \\
\mathbb{Z}^{4} & \text { if } i=3 \\
0 & \text { if } i=4 \\
(\mathbb{Z} / 2)^{3} & \text { if } i=5 \\
\mathbb{Z}^{6} & \text { if } i=6 \\
0 & \text { if } i \geq 7 .
\end{array}\right.\right.
$$

It turns out that there is a single non-zero differential on the $E_{2}$ page, namely $d_{2}$ : $E_{2}^{0,2} \rightarrow E_{2}^{2,1}$, which is an isomorphism. This differential is forced by the fact that $X[3]$ is simply connected. There are no other non-trivial differentials: the only possible one would be $E_{2}^{0,5} \rightarrow E_{2}^{2,4}$; however these terms necessarily appear in the cohomology of $X[3]$ as through duality the term $E_{2}^{0,5}$ represents the top cohomology of the three copies of the top pieces of $\operatorname{Hom}\left(\mathbb{Z}^{2}, S U(2)\right)$ which must split off stably from $\operatorname{Hom}\left(\mathbb{Z}^{3}, S U(2)\right)$. Using this we can obtain a corrected version of Theorem 5.4 in [1]: 


$$
H_{i}(X[3], \mathbb{Z}) \cong \begin{cases}\mathbb{Z} & \text { if } i=0,1,2 \\ \mathbb{Z}^{4} & \text { if } i=3 \\ (\mathbb{Z} / 2)^{4} & \text { if } i=4 \\ \mathbb{Z} / 2 & \text { if } i=5 \\ \mathbb{Z}^{3} & \text { if } i=6 \\ 0 & \text { if } i \geq 7 .\end{cases}
$$

Applying Poincaré-Lefschetz duality to the pair $\left(S U(2)^{3}, \operatorname{Hom}\left(\mathbb{Z}^{3}, S U(2)\right)\right)$ now yields the revised calculation:

Theorem 0.1 The integral cohomology of the space of (ordered) commuting triples in $S U(2)$ is given by

$$
H^{i}\left(\operatorname{Hom}\left(\mathbb{Z}^{3}, S U(2)\right), \mathbb{Z}\right) \cong \begin{cases}\mathbb{Z} & \text { if } i=0 \\ 0 & \text { if } i=1 \\ \mathbb{Z}^{3} & \text { if } i=2 \\ \mathbb{Z}^{3} \oplus \mathbb{Z} / 2 & \text { if } i=3 \\ (\mathbb{Z} / 2)^{4} & \text { if } i=4 \\ \mathbb{Z} & \text { if } i=5 \\ 0 & \text { if } i \geq 6 .\end{cases}
$$

This agrees with the results implied in Crabb's paper, and is thus a corrected version of Theorem 1.4 in [1]. As a consequence of these corrections, our identification of $\operatorname{Hom}\left(\mathbb{Z}^{3}, S U(3)\right) / S_{3}(S U(2))$ with $S U(2) \wedge\left(\mathbb{S}^{6}-S O(3)\right)$ must be modified; as shown by $\mathrm{Crabb}$ this piece is in fact homotopy equivalent to the stunted projective space $\mathbb{R} P^{5} / \mathbb{R} P^{3}$. The corresponding modification must be made in Examples 6.9 and 6.10 .

Similarly there is a mistake in the calculation for the cohomology of $\operatorname{Hom}(\mathbb{Z} \times$ $F_{2}, S U(2)$ ) (Proposition 4.11) where $F_{2}$ is the free group on two generators. The cohomology of the space $S U(2)^{3}-\operatorname{Hom}\left(\mathbb{Z} \times F_{2}, S U(2)\right)$ is actually given by

$$
H^{i}\left(S U(2)^{3}-\operatorname{Hom}\left(\mathbb{Z} \times F_{2}, S U(2)\right), \mathbb{Z}\right) \cong \begin{cases}\mathbb{Z} & \text { if } i=0 \\ 0 & \text { if } i=1 \\ \mathbb{Z} & \text { if } i=2 \\ \mathbb{Z}^{3} & \text { if } i=3 \\ 0 & \text { if } i=4 \\ \mathbb{Z} \oplus \mathbb{Z} / 2 \oplus \mathbb{Z} / 2 & \text { if } i=5 \\ \mathbb{Z} \oplus \mathbb{Z} & \text { if } i=6 \\ 0 & \text { if } i \geq 7 .\end{cases}
$$

A differential in the spectral sequence used to calculate this was overlooked. Using duality as explained in [1] this leads to the corrected version of Theorem 4.12: 
Theorem 0.2 The integral cohomology of $\operatorname{Hom}\left(\mathbb{Z} \times F_{2}, S U(2)\right)$ is given by

$$
H^{i}\left(\operatorname{Hom}\left(\mathbb{Z} \times F_{2}, S U(2)\right), \mathbb{Z}\right) \cong \begin{cases}\mathbb{Z} & \text { if } i=0 \\ 0 & \text { if } i=1 \\ \mathbb{Z}^{2} & \text { if } i=2 \\ \mathbb{Z}^{4} & \text { if } i=3 \\ (\mathbb{Z} / 2)^{2} & \text { if } i=4 \\ \mathbb{Z} & \text { if } i=5 \\ \mathbb{Z} \oplus \mathbb{Z} & \text { if } i=6 \\ 0 & \text { if } i \geq 7 .\end{cases}
$$

From this analysis it also follows that the corresponding claim (Theorem 4.13) made about the cohomology of $\operatorname{Hom}\left(\mathbb{Z} \times F_{r}, S U(2)\right)$ where $F_{r}$ is the free group on $r$ generators is incorrect; however the correct calculation can be obtained from [2].

We also take this opportunity to correct a typo: in the proof for Proposition 3.1 the correct value for $H_{1}\left(\Gamma_{2}, \mathbb{Z}\right)$ is $\mathbb{Z} / 10$, not $\mathbb{Z} / 20$.

We are grateful to M. Crabb for his interest in our work as well as pointing out the above mistakes in our cohomology calculations. The rest of the paper is unaffected.

\section{References}

1. Adem, A., Cohen, F.: Commuting elements and spaces of homomorphisms. Math. Ann. 338, 587-626

2. Crabb, M.: Spaces of commuting elements in $S U(2)$, preprint 2008 